\title{
IMMEDIATE AND VIRTUAL BASINS OF NEWTON'S METHOD FOR ENTIRE FUNCTIONS
}

\author{
SEBASTIAN MAYER, DIERK SCHLEICHER
}

\begin{abstract}
We investigate the well known Newton method to find roots of entire holomorphic functions. Our main result is that the immediate basin of attraction for every root is simply connected and unbounded. We also introduce "virtual immediate basins" in which the dynamics converges to infinity; we prove that these are simply connected as well.
\end{abstract}

\section{IntRoduction}

Newton's method is one of the preferred methods to find roots of differentiable maps: it often converges very fast and it is very easy to implement. But there are problems: for example, even for polynomials, there are open sets of initial conditions for which the Newton map does not converge to any root.

There has been substantial progress understanding the dynamics of Newton's method for finding roots of complex polynomials: Przytycki $[\mathrm{P}]$ has shown that all immediate basins are simply connected and unbounded; Shishikura Sh has shown more generally that if a rational map has a multiply connected Fatou component, then it must have two repelling or parabolic fixed points (which is impossible for Newton maps of polynomials). Hubbard, Schleicher and Sutherland [HSS] used this to find a rather small set of starting points which together find all roots of a complex polynomial; and in [Sch] there is a (not very efficient) bound on the number of iterations it takes to find all roots with given accuracy. In a different spirit, Smale [Sm has shown that Newton's method is quite efficient from a probabilistic point of view.

Newton's method for transcendental entire functions is less understood. Bergweiler and Terglane [BT] have shown that Newton maps have no multiply connected wandering domains, and in certain cases no wandering domains at all.

In this note, we extend Przytycki's result to entire holomorphic functions: for every root $\xi$ of a non-constant entire holomorphic function $f$, the immediate basin of attraction for the Newton map associated to $f$ is simply connected and unbounded. This result goes back to the Diploma thesis [Ma. While the result and part of its proof are in analogy to the polynomial case, it turns out that for entire holomorphic functions, but not for polynomials, "virtual immediate basins" play

Date: August 11, 2018.

2000 Mathematics Subject Classification. 30D05,37F10,37N30.

Key words and phrases. Newton method, entire function, immediate basin, virtual basin. 
an important role: these are domains in which the dynamics converges to $\infty$ as if there was a root at $\infty$ (subject to further conditions; see Definition 3.2). We show also that every virtual immediate basin is simply connected. The combinatorial restrictions on ordinary and virtual immediate basins are investigated in [MRS].

It would be interesting to extend the ideas of [Sh] to the transcendental case, showing that all Fatou components are simply connected; the case of wandering domains is treated in $[\mathrm{BT}]$.

Acknowledgements. We would like to thank Johannes Rückert for his many helpful comments. We are grateful for the hospitality and the constructive atmosphere at the Institut Henri Poincaré, Université Paris VI.

\section{IMmediate BASINS}

Throughout this paper, let $f: \mathbb{C} \rightarrow \mathbb{C}$ be a nonlinear entire holomorphic map and $N_{f}=$ id $-f / f^{\prime}$ its associated Newton map. We will be concerned with the set of points which converge to any given root $\xi$ of $f$. Clearly, the roots of $f$ are exactly the fixed points of $N_{f}$ in $\mathbb{C}$, and these are attracting.

Definition 2.1 (Immediate basin).

Let $\xi$ be an attracting fixed point of $N_{f}$. The basin of attraction of $\xi$ is the open set of all points $z$ such that $\left(N_{f}^{\circ m}(z)\right)_{m \in \mathbb{N}}$ converges to $\xi$. The connected component containing $\xi$ of the basin is called the immediate basin of $\xi$.

Throughout this paper, we will fix a root $\xi$ of $f$ and denote its immediate basin by $U$. In order to show that $U$ is both simply connected and unbounded, we will construct a curve $\gamma: \mathbb{R}_{0}^{+} \rightarrow U$ with $N_{f}(\gamma(t))=\gamma(t-1)$ for $t \geq 1$. If $U$ fails to be full, then we can arrange things so that $\gamma\left(\mathbb{R}_{0}^{+}\right)$is bounded (and the same is obviously true if $U$ itself is bounded). In this case, we show that $\lim _{t \rightarrow+\infty} \gamma(t)$ is a fixed point of $N_{f}$ in $\partial U \cap \mathbb{C}$, and this will lead to a contradiction.

2.1. An Exhaustion of Immediate Basins. Let Crit $\left(N_{f}\right)$ be the set of critical values of $N_{f}$ and

$$
P_{U}:=\bigcup_{m \geq 0} N_{f}^{\circ m}\left(\operatorname{Crit}\left(N_{f}\right) \cap U\right)
$$

be the postcritical set restricted to critical values in $U$. Since $\operatorname{Crit}\left(N_{f}\right)$ is countable, the set $P_{U}$ is countable as well (but in general not closed). There is thus an open disk $S_{0}=B_{r}(\xi) \subset U$ centered at $\xi$ such that $\partial S_{0} \cap P_{U}=\emptyset$, and small enough such that $N_{f}\left(\overline{S_{0}}\right) \subset S_{0}$. For every $k \in \mathbb{N}_{0}$ define $S_{k+1}$ to be the connected component of $N_{f}^{-1}\left(S_{k}\right)$ containing $S_{0}$; then $\overline{S_{k+1}}$ is the connected component of $N_{f}^{-1}\left(\overline{S_{k}}\right)$ containing $S_{0}$.

Lemma 2.2. The immediate basin satisfies $U=\bigcup_{k \in \mathbb{N}} S_{k}$. 
Proof. Clearly, $U$ is open and $U^{\prime}:=\bigcup_{k \in \mathbb{N}} S_{k}$ is an open subset of $U$. Suppose there is a $z \in U \backslash U^{\prime}$. Then there is an $M \in \mathbb{N}$ with $N_{f}^{\circ M}(z) \in S_{0}$, so there is a connected neighborhood $V \subset U$ of $z$ with $N_{f}^{\circ M}(V) \subset S_{0}$. For all $m \geq M$, z is by assumption in a component of $N_{f}^{-m}\left(S_{0}\right)$ different from $S_{m}$, and so is $V$. Hence $U \backslash U^{\prime}$ is open in contradiction to the fact that $U$ is connected.

Recall that a subset of $\mathbb{C}$ is called full if its complement has no bounded components. Clearly, if $U$ is not full, then some of the $S_{k}$ are not full (if $U$ is not full, then it contains a non-contractible loop which is compact and thus contained in finitely many $S_{k}$ ).

Lemma 2.3 (Bounded $C_{k}$ ). If $S_{M}$ is not full but $S_{M-1}$ is, then all $S_{m}$ with $m<M$ are bounded and homeomorphic to open disks.

Proof. There is a bounded connected component $B$ of $\mathbb{C} \backslash S_{M}$. Its boundary $\partial B$ is a compact subset of $\overline{S_{M}}$, so $N_{f}(\partial B)$ is a compact subset of $\overline{S_{M-1}}$. There are no postcritical points in $\partial S_{0}$, so $N_{f}^{\circ M}$ restricted to a neighborhood of $\partial B$ is a local injection and $N_{f}^{\circ M}: \partial B \rightarrow \partial S_{0}$ is a covering map. This implies that $\partial B$ and $N_{f}(\partial B)$ are homeomorphic to circles. Since $N_{f}(\partial B)$ is a boundary component of $S_{M-1}$ and $S_{M-1}$ is full, it follows that $S_{M-1}$ is contained in the bounded complementary component of $N_{f}(\partial B)$, so $S_{M-1}$ is bounded and homeomorphic to an open disk.

Clearly, all $S_{m}$ with $m<M$ are contained in $S_{M-1}$, hence also bounded and simply connected.

2.2. Extending Paths Invariantly to Infinity. The goal of this section is the construction of curves $\delta: \mathbb{R}_{0}^{+} \rightarrow U$ such that $N_{f}(\delta(t))=\delta(t-1)$ for $t \geq 1$.

Definition 2.4 (Extension of a curve). If $\delta:[0,1] \rightarrow \mathbb{C} \backslash \operatorname{Crit}\left(N_{f}\right)$ is a curve with $N_{f}(\delta(1))=\delta(0)$, then define its (maximal) extension $\operatorname{ext}_{\delta}:\left[0, M_{\delta}\right) \rightarrow \mathbb{C} \backslash \operatorname{Crit}\left(N_{f}\right)$ to be the unique curve with

$$
\begin{gathered}
\forall t \in\left[0, M_{\delta}-1\right): \quad N_{f}\left(\operatorname{ext}_{\delta}(t+1)\right)=\operatorname{ext}_{\delta}(t) \\
\left.\operatorname{ext}_{\delta}\right|_{[0,1]} \equiv \delta
\end{gathered}
$$

where $M_{\delta}$ is chosen maximal in $[1, \infty) \cup\{\infty\}$.

Lemma 2.5 (Possibilities for the extension of a curve).

Given any curve $\delta:[0,1] \rightarrow \mathbb{C} \backslash \operatorname{Crit}\left(N_{f}\right)$, then exactly one of three cases occurs: (i) $M_{\delta}=\infty$, (ii) $M_{\delta}<\infty$ and $\lim _{t \rightarrow M_{\delta}}$ ext $t_{\delta}(t)$ is a critical point of $N_{f}$, or (iii) $M_{\delta}<\infty, \operatorname{ext}_{\delta}\left(M_{\delta}-1\right)$ is an asymptotic value, and $\operatorname{ext}_{\delta}(t) \rightarrow \infty$ as $t \rightarrow M_{\delta}$.

Proof. Choose $T \in\left(0, M_{\delta}\right]$ with $T<\infty$ and suppose there is a sequence $t_{n} \nearrow T$ with $\operatorname{ext}_{\delta}\left(t_{n}\right) \rightarrow a \in \mathbb{C}$. If $a$ is not a critical point, then there is a neighborhood $V$ of $a$ such that $\left.N_{f}\right|_{V}$ is univalent, and it follows that $T<M_{\delta}$. Therefore, if $M_{\delta}<\infty$, then $\operatorname{ext}_{\delta}$ converges either to a critical point or to infinity along an asymptotic path. 
The following lemma is related to typical proofs of "landing of periodic dynamic rays" for iterated polynomials.

Lemma 2.6 (A homotopy class of unbounded curves).

Let $W, W^{\prime} \subset U \backslash S_{0}$ be two simply connected domains such that $N_{f}\left(W^{\prime}\right) \subset W$ and $W^{\prime} \subset W$. Let

$$
S_{W^{\prime}, W}:=\left\{\sigma:[0,1] \rightarrow W \text { continuous, } \sigma(1) \in W^{\prime}, \sigma(0)=N_{f}(\sigma(1))\right\} .
$$

If there is an extension ext $\sin _{\sigma}[0, \infty) \rightarrow \mathbb{C} \backslash \operatorname{Crit}\left(N_{f}\right)$ for all $\sigma \in S_{W, W^{\prime}}$, then every $\sigma \in S_{W, W^{\prime}}$ has $\lim _{t \rightarrow \infty} \operatorname{ext}_{\sigma}(t)=\infty$.

Proof. Set $Y:=\bigcup_{\sigma \in S_{W, W^{\prime}}}$ image $\left(\operatorname{ext}_{\sigma}\right)$. We use $S_{W^{\prime}, W}$ to construct a sequence of local inverse mappings of $N_{f}^{o m}$ on $W$. Note that the hypothesis implies that $W \cap \operatorname{Crit}\left(N_{f}\right)=\emptyset$.

For $n \in \mathbb{N}$, we define maps $\eta_{n}: W \longrightarrow Y$ as follows: given $z \in W$, choose a curve $\sigma \in S_{W^{\prime}, W}$ and $0 \leq t \leq 1$ such that $\sigma(t)=z$ and define $\eta_{n}(z)=\operatorname{ext}_{\sigma}(t+n)$. It is easy to check that this is well-defined, i.e. independent of the choice of curve and its parametrization. The maps $\eta_{n}$ are continuous for all $n \in \mathbb{N}$ with $\eta_{n}^{-1}=N_{f}^{o n}$, so all $\eta_{n}$ are holomorphic. The sequence $\left(\eta_{n}\right)_{n \in \mathbb{N}}$ clearly forms a normal family, so there is a locally uniformly convergent subsequence $\left(\eta_{n_{l}}\right)_{l \in \mathbb{N}}$; its limit function $\eta: W \rightarrow \bar{Y}$ is holomorphic by the theorem of Weierstraß.

Suppose there is a $y_{0} \in \operatorname{image}(\eta) \cap \mathbb{C}$. We have $W \cap S_{0}=\emptyset$, so for all $m \in \mathbb{N}$ and sufficiently big $n$ (depending on $m$ ), image $\left(\eta_{n}\right) \cap S_{m}=\emptyset$. Furthermore $U=\bigcup_{m \geq 0} S_{m}$ is open, so $y_{0} \in \partial U$ and by Hurwitz' theorem, $\eta \equiv y_{0}$ is constant. If $y_{0} \neq \infty$, then choose some $w \in W^{\prime}$ and define $z:=N_{f}(w)$. Then

$$
N_{f}\left(y_{0}\right)=N_{f}(\eta(w))=\lim _{l \rightarrow \infty} N_{f}\left(\eta_{n_{l}}(w)\right)=\lim _{l \rightarrow \infty} \eta_{n_{l}}(z)=\eta(z)=y_{0} .
$$

Thus $y_{0}$ is a fixed point of $N_{f}$ on $\partial U \cap \mathbb{C}$, while the only fixed points of $N_{f}$ in $\mathbb{C}$ are the zeros of $f$, and these are not on the boundary of $U$. This contradiction shows that $y_{0}=\infty$.

Suppose there is a single curve $\sigma \in S_{W, W^{\prime}}$ for which $\lim _{t \rightarrow \infty} \operatorname{ext}_{\sigma}(t)=\infty$ is false. Then there is a subsequence $\left(\eta_{n_{l}}\right)$ for which the limit function $\eta: W \rightarrow \bar{Y}$ could not be identically equal to $\infty$, and this is a contradiction which proves the lemma.

REMARK. A different way to prove this lemma is to use contraction properties of the hyperbolic metric in $U \backslash \overline{P_{U}}$.

2.3. Immediate Basins are Simply Connected and Unbounded. Now it is time for our first main result.

Theorem 2.7 (Immediate basins).

Let $f: \mathbb{C} \rightarrow \mathbb{C}$ be a nonlinear entire map, $N_{f}:=i d-\frac{f}{f^{\prime}}$ its Newton map and $\xi$ a root of $f$. Then the immediate basin $U$ of $\xi$ is simply connected and unbounded. 
Proof. Choose an open disk $S_{0}$ around $\xi$ with $N_{f}\left(\overline{S_{0}}\right) \subset S_{0}, \partial S_{0} \cap P_{U}=\emptyset$ and define $S_{k+1}$ as the component of $N_{f}^{-1}\left(S_{k}\right)$ containing $S_{0}$. Note that $N_{f}$ has no postcritical points on $\partial S_{0}$, so there are no critical points on any $\partial S_{k}$ and $N_{f}$ is locally biholomorphic in a neighborhood of $\partial S_{k}$.

By Lemma 2.2. $U=\bigcup_{n \geq 0} S_{n}$. If $U$ is not simply connected, then there is a minimal $M$ such that $S_{M}$ is not full. Choose a bounded component $B_{0}$ of $\mathbb{C} \backslash S_{M}$; then there is a bounded component $B$ of $U \backslash S_{M}$ as well. If $U$ is bounded, let $B:=U \backslash S_{M}$ for an arbitrary $M$. In both cases, $B$ is a bounded component of $U \backslash S_{M}$, and this will lead to a contradiction (compare Figure 田).

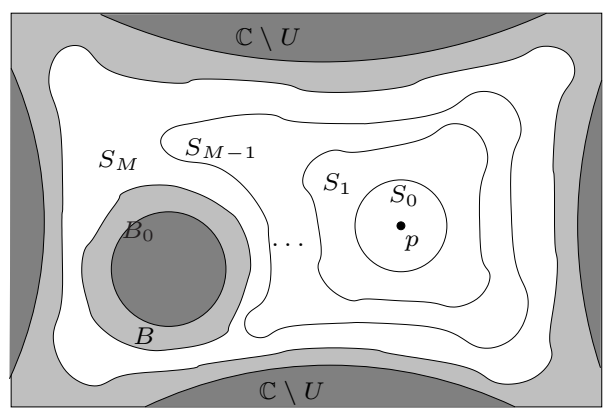

Figure 1. $U=$ $\bigcup_{n \geq 0} S_{n}$ and $B$

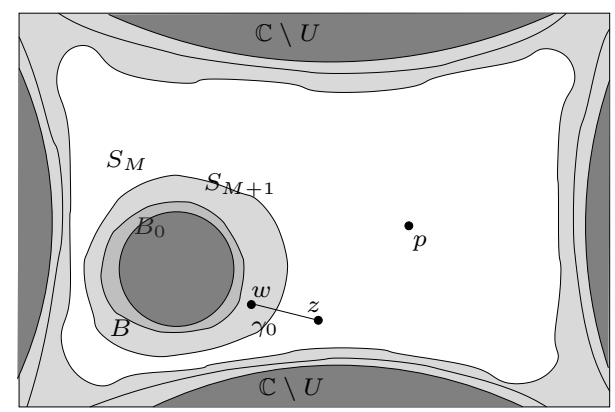

Figure 2. How to choose $\gamma^{0}$.

Define $P_{B}:=\bigcup_{m \geq 0} N_{f}^{o m}\left(\operatorname{Crit}\left(N_{f}\right) \cap B\right)$; since $B$ is bounded, $\operatorname{Crit}\left(N_{f}\right) \cap B$ is finite, and the only accumulation point of $P_{B}$ is $\xi$. Choose $w \in\left(S_{M+1} \backslash \overline{S_{M}}\right) \cap$ $B \backslash P_{B}$ (compare Figure 2) and set $z:=N_{f}(w) \in S_{M} \backslash \overline{S_{M-1}}$. There is an injective path $\gamma^{0}:[0,1] \rightarrow\left(S_{M+1} \backslash \overline{S_{M-1}}\right) \backslash P_{B}$ with $\gamma^{0}(0)=z$ and $\gamma^{0}(1)=w$.

We want to show that $\operatorname{ext}_{\gamma^{0}}$ converges to $\infty$ within $B$, which would be a contradiction. Since $\gamma^{0}([0,1]) \cap P_{B}=\emptyset$, there is a maximal curve $\gamma:=\operatorname{ext}_{\gamma^{0}}$ : $\left[0, M_{\gamma}\right) \rightarrow \mathbb{C}$ with:

$$
\begin{aligned}
\forall t \in\left[1, M_{\gamma}\right): \quad N_{f}(\gamma(t)) & =\gamma(t-1) \\
\left.\gamma\right|_{[0,1]} & \equiv \gamma^{0} .
\end{aligned}
$$

By induction, it follows that $\gamma([n, n+1]) \subset S_{M+n+1} \backslash \overline{S_{M+n-1}}$ for every $n \in \mathbb{N}$, and in particular we have $\gamma\left(\left[1, M_{\gamma}\right)\right) \subset U \backslash \overline{S_{M}}$. In fact, even $\gamma\left(\left[1, M_{\gamma}\right)\right) \subset B$ (because $B$ is the component of $U \backslash S_{M}$ containing $\gamma(1)=w$ ). Since $B$ is bounded, Lemma 2.5 implies that $M_{\gamma}=\infty$.

Choose an open, bounded and simply connected neighborhood $W \subset S_{M+1} \backslash$ $\overline{S_{M-1}}$ of image $\left(\gamma^{0}\right)$ disjoint from $P_{B}$. This can be done because image $\left(\gamma^{0}\right)$ and $\bar{P}_{B}=P_{B} \cup\{\xi\}$ are compact and disjoint. 


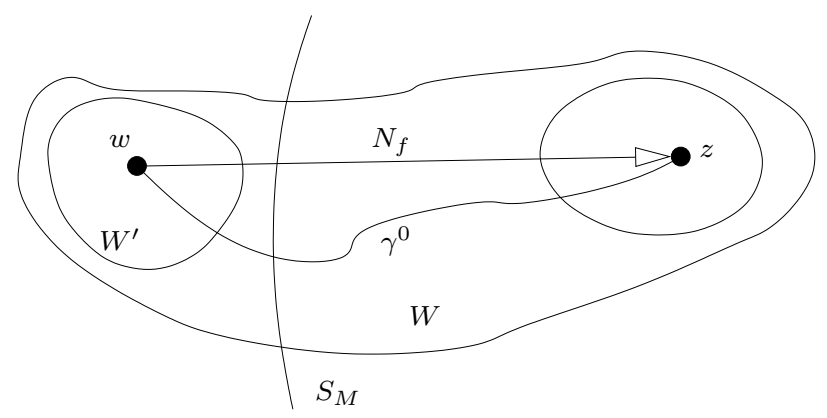

FiguRE $3 . W$ and $W^{\prime}$.

Let $W^{\prime} \subset W$ be a simply connected neighborhood of $w$ with $N_{f}\left(W^{\prime}\right) \subset W$ (compare Figure 31). By Lemma 2.5. every curve

$$
\sigma \in S:=\left\{\sigma:[0,1] \rightarrow W \text { continuous, } \sigma(1) \in W^{\prime}, \sigma(0)=N_{f}(\sigma(1))\right\}
$$

has an extension $\operatorname{ext}_{\sigma}:[0, \infty) \rightarrow B \cup W$. By Lemma 2.6. every curve $\sigma \in S$ satisfies $\lim _{t \rightarrow \infty} \operatorname{ext}_{\sigma}(t)=\infty$, so $B$ is unbounded: a contradiction.

REMARK. In many cases, it even follows that $\infty$ is accessible within $U$. The case in which we cannot prove this is if $U$ contains infinitely many critical points of $N_{f}$ such that $P_{U}$ is dense in $U$.

\section{Virtual Immediate Basins}

3.1. A Motivating Example. The dynamics of Newton's map for transcendental entire functions has a class of Fatou components which we want to call virtual immediate basins. Let $f(z):=z \exp \left(-\frac{1}{n} z^{n}\right)$; its Newton map $N_{f}(z)=$ $z\left(1-\frac{1}{1-z^{n}}\right)$ s a rational function. The involution $\iota: \hat{\mathbb{C}} \rightarrow \hat{\mathbb{C}}, z \mapsto \frac{1}{z}$ conjugates $N_{f}$ to the polynomial $\iota \circ N_{f} \circ \iota(\zeta)=\zeta-\zeta^{n+1}$. In this case, the Leau-Fatou "Flower Theorem" shows that there are exactly $n$ attracting and $n$ repelling petals at $\zeta=0$, so $N_{f}$ has exactly $n$ unbounded Fatou components with convergence to $z=\infty$; moreover, the immediate basin of the root 0 has exactly $n$ accesses to $\infty$ (these accesses are called "channels to $\infty$ " [HSS]); compare Figure 4 We call the attracting petals at infinity virtual immediate basins: their dynamics is similar as if there was a root at $\infty$ in each of these $n$ directions. Note that the $n$ channels of the root 0 separate all these $n$ virtual basins.

For Newton's method of polynomials, it is known that any pair of channels to $\infty$ of the same root must enclose a different root of the polynomial. As this example shows, an analogous statement for transcendental entire functions would be false if virtual immediate basins were not taken into account. An investigation of the combinatorial possibilities between channels to $\infty$ and immediate basins (including virtual basins) can be found in [MRS. 

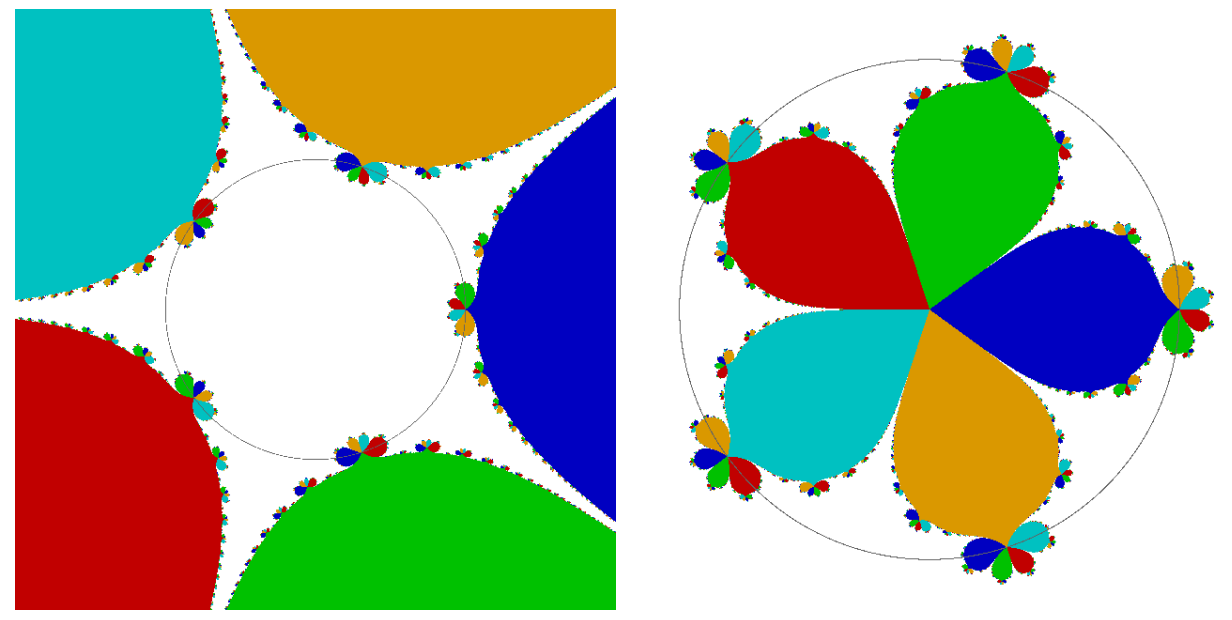

Figure 4. Dynamics of the Newton for $f(z)=z e^{-\frac{1}{5} z^{5}}$. The immediate basin of the root 0 is white, the other colors correspond to virtual immediate basins and their backward images. The unit circle $\mathbb{S}^{1}$ is marked in grey. Right: The same situation in $\zeta=\iota(z)=1 / z$ coordinates: $\iota \circ N_{f} \circ \iota^{-1}(\zeta)=\zeta-\zeta^{n+1}$ is a polynomial.

3.2. An Exhaustion of Virtual Immediate Basins. In order to define virtual immediate basins, we need the following definition.

Definition 3.1 (Absorbing set). If $U$ is an $N_{f}$-invariant domain in $\mathbb{C}$, then an open set $A \subset \mathbb{C}$ is called absorbing set (of $U$ ) if the following conditions hold:

(1) $A$ is simply connected and $\bar{A}$ is full;

(2) $\overline{N_{f}(A)} \subset A$;

(3) for every $z \in U$ there is a $k$ such that $N_{f}^{\circ k}(z) \in A$.

Definition 3.2 (Virtual immediate basin). A domain $U \subset \mathbb{C}$ is called a virtual immediate basin if it is maximal with respect to the following properties:

(1) $\lim _{n \rightarrow \infty} N_{f}^{o n}(z)=\infty$ for all $z \in U$

(2) there is an absorbing set $A \subset U$.

Virtual immediate basins occur only for Newton maps of transcendental entire functions. Obviously, every virtual immediate basin is unbounded with $\infty$ as accessible boundary point, and the same is true for every absorbing set of a virtual immediate basin. If $N_{f}$ is rational (as in our example above), then a virtual immediate basin is a Leau-Fatou petal; otherwise, it is (contained in) a "Baker domain" (a domain at $\infty$ ); see below.

Let $U$ be a virtual immediate basin. By a small modification of the absorbing set, we find an absorbing set $S_{0}$ of $U$ such that $\partial S_{0}$ contains no postcritical points. Similarly as for immediate basins, define $S_{k+1}$ to be the connected component of $N^{-1}\left(S_{k}\right)$ containing $S_{0}$, for all $k \geq 0$. As before, we have the following: 
Lemma 3.3. $U$ is open and $U=\bigcup_{k \in \mathbb{N}} S_{k}$. If $U$ is not full, then one of the $S_{k}$ is not full.

3.3. Simple Connectivity. In a number of ways, virtual immediate basins have similar properties as immediate basins of roots; here is one such result.

Theorem 3.4. Virtual immediate basins are simply connected.

Proof. Let $U$ be a virtual immediate basin with absorbing set $S_{0}$. By Lemma 3.3 there is an exhaustion $U=\bigcup_{m \in \mathbb{N}} S_{m}$ of $U$. If $U$ is not full, then there is a minimal $M$ such that $S_{M}$ is not full.

There is a bounded connected component $B$ of $\mathbb{C} \backslash S_{M}$. Its boundary $\partial B$ is a compact subset of $S_{M}$, so $N_{f}(\partial B)$ is a compact subset of $S_{M-1}$.

There are no critical points in $\partial S_{M} \cup \partial S_{M-1} \cup \ldots \cup \partial S_{0}$, so $N_{f}^{M}$ maps $\partial B$ onto a component of $\partial S_{0}$. Since $\partial B$ is a compact subset of $\mathbb{C}$, so is $\partial S_{0}$. But $S_{0} \subset \mathbb{C}$ is unbounded and simply connected, so it follows that $\partial S_{0}$ is unbounded as well, and this is a contradiction.

We cannot show in general that every virtual immediate basin equals an entire Fatou component; however, we have the following.

Remark 3.5. Every virtual immediate basin $U$ is contained in an invariant Fatou component $F$. If $F$ is simply connected and $\left.N\right|_{F}$ is a proper map, then $F$ is a virtual immediate basin.

This simply follows by using a Riemann map $\varphi: F \rightarrow \mathbb{D}$ to transport the dynamics of $F$ into the unit disk $\mathbb{D}$. It might be possible to extend Shishikura's results [Sh] to the transcendental case, to show the more general result that every Fatou component of Newton's map for entire functions is simply connected. In this case, every virtual immediate basin would be an entire Fatou component provided the Newton dynamics restricted to this Fatou component was proper.

\section{REFERENCES}

[BT] Walter Bergweiler, Norbert Terglane: Weakly repelling fixpoints and the connectivity of wandering domains, Transactions of the American Mathematical Society, 3481 (1996), $1-12$.

[HSS] John Hubbard, Dierk Schleicher, Scott Sutherland: How to find All Roots of complex Polynomials by Newton's Method Inventiones Mathematicae 146 (2001), 1-33.

[Ma] Sebastian Mayer: Newton's method for entire functions, Diplomarbeit, Technische Universität München (2002).

[MRS] Sebastian Mayer, Johannes Rückert, Dierk Schleicher: Combinatorial structure of immediate basins of Newton maps. Manuscript, in preparation.

[P] Feliks Przytycki: Remarks on the simple connectedness of basins of sinks for iterations of rational maps. In: Dynamical Systems and Ergodic Theory, ed. K. Krzyzewski. Polish Scientific Publishers, Warszawa (1989), 229-235.

[Sch] Dierk Schleicher: On the number of iterations of Newton's method for complex polynomials, Ergodic Theory Dyn. Syst. 223 (2002), 935-945. 
[Sh] Mitsuhiro Shishikura: The connectivity of the Julia set and fixed points, Preprint IHES 37 (1990).

[Sm] Steven Smale, On the efficiency of algorithms of analysis, Bulletin of the American Mathematical Society 132 (1985), 87-121.

Lehrstuhl A für Mathematik, RWTH Aachen, D-52056 Aachen, Germany

E-mail address: sebastian.mayer@mathA.rwth-aachen.de

School of Engineering and Science, International University Bremen, PostFaCh 750 561, D-28725 Bremen, Germany

E-mail address: dierk@iu-bremen.de 